\title{
Drugs to Foster Kidney Regeneration in Experimental Animals and Humans
}

\author{
Elena Gagliardini Ariela Benigni \\ IRCCS - Istituto di Ricerche Farmacologiche 'Mario Negri', Centro Anna Maria Astori, Science and Technology Park \\ Kilometro Rosso, Bergamo, Italy
}

\section{Key Words}

Kidney regeneration - Angiotensin-converting enzyme inhibitors · Renal progenitors

\begin{abstract}
Background: The incidence of kidney diseases is increasing worldwide and they are emerging as a major public health problem. Once mostly considered inexorable, renal disease progression can now be halted and lesions can even regress with drugs such as angiotensin-converting enzyme inhibitors (ACEi) and angiotensin II type I receptor blockers, indicating the possibility of kidney repair. Summary: The discovery of renal progenitor cells lining the Bowman capsule of adult rat and human kidneys has shed light on the mechanism of repair by ACEi. Parietal progenitors are a reservoir of cells that contribute to podocyte turnover in physiological conditions. In the early phases of renal disease these progenitors migrate chaotically and subsequently proliferate, accumulating in Bowman's space. The abnormal behavior of parietal progenitors is sustained by the activation of CXCR4 receptors in response to an increased production of the chemokine SDF- 1 by podocytes activated by the inflammatory environment. Ang II, via the $\mathrm{AT}_{1}$ receptor, also contributes to progenitor cell proliferation. The CXCR4/SDF-1 and Ang II/ $A T_{1}$ receptor pathogenic pathways both pave the way for le-
\end{abstract}

sion formation and subsequent sclerosis. ACEi normalize the CXCR4 and $A T_{1}$ receptor expression on progenitors, limiting their proliferation, concomitant with the regression of hyperplastic lesions in animals, and in a patient with crescentic glomerulopathy. Key Message: Understanding the molecular and cellular determinants of regeneration triggered by renoprotective drugs will reveal novel pathways that might be challenged or targeted by pharmacological therapy.

(c) 2014 S. Karger AG, Basel

\section{Introduction}

Chronic kidney diseases (CKD) are a worldwide threat to public health. It has been estimated that diseases of the kidney account for 830,000 deaths every year, with the sharp rise of renal replacement therapy now exceeding 2 million patients for a cost of more than USD 1 trillion [1]. The burden of CKD is not limited to the demand for renal replacement therapies, since they are also a major determinant of cardiovascular diseases, with a direct impact on health [2]. Limiting the progression of kidney disease by lowering blood pressure and the levels of urinary proteins also translates into protection of patients from cardiovascular complications [3].

\section{KARGER}

E-Mail karger@karger.com

www.karger.com/nee
(C) 2014 S. Karger AG, Basel

$1660-2129 / 14 / 1262-0091 \$ 39.50 / 0$
Ariela Benigni, $\mathrm{PhD}$

IRCCS - Istituto di Ricerche Farmacologiche 'Mario Negri'

Centro Anna Maria Astori, Science and Technology Park Kilometro Rosso

Via Stezzano 87, IT-24126 Bergamo (Italy)

E-Mail ariela.benigni@ marionegri.it 
Renal outcomes are mostly unfavorable in patients with progressive kidney disease, showing the kidney's limited capacity to repair chronic damage. However, experimental and clinical evidence indicates that there are drugs that can interfere with the tendency to progress to end-organ damage, offering new hope for the future of kidney regeneration.

\section{Kidney Repair in Animals}

Renal disease progression is sustained by common mechanisms, starting with nephron loss, which lead to a process of adaptation of the remaining nephrons including glomerular hypertension and enlargement of the pores perforating the glomerular barrier, with a consequent loss of size-selective properties. The resulting excessive concentration of ultrafiltered proteins accumulating in Bowman's space and in the lumen of tubules contributes to further damage through the activation of inflammatory and apoptotic pathways fuelling progressive renal damage [4].

Drugs that reduce glomerular hypertension and protein trafficking limit renal function decline and promote kidney repair. The cornerstone of the current treatment is inhibition of the renin-angiotensin system (RAS) by angiotensin-converting enzyme inhibitors (ACEi) and angiotensin II type I receptor blockers (ARBs). Animal models of nondiabetic and diabetic nephropathy have clearly shown that treatment with ACEi and ARBs or their combination not only prevents progressive renal damage but also promotes the regression of glomerulosclerosis and vascular lesions [5].

Three-dimensional reconstruction of the glomerular capillary tuft [6] based on serial section analysis of the kidney has helped to show that ACEi reduces the glomerular volume occupied by sclerosis. In Munich-WistarFromter (MWF) rats (which are genetically programmed to develop renal injury with age) studied at a stage of advanced nephropathy, high-dose ACEi reduced the volume of sclerosis in most glomeruli in parallel with a substantial increase in the volume occupied by capillaries [6], suggesting remodeling of the glomerular architecture and regeneration of the capillary network. The regression of glomerulosclerosis was attributed to the effect of angiotensin II antagonism of reducing extracellular matrix deposition through reduced expressions of plasminogen activator inhibitor-1 [7], collagen I and IV, and transforming growth factor- $\beta[6]$ and increased metalloproteinase activity [8]. The resolution of glomerular lesions could be attributed to an effect of ACEi on the modulation of survival and repair by glomerular cells themselves or progenitor cells possibly populating the kidney.

Previous data described the regeneration of glomerular capillaries as a result of glomerular cell repopulation after ACEi. Enalapril at high doses in rats with subtotal nephrectomy limited mesangial cell proliferation and normalized the number of endothelial cells [9]. More recent evidence indicated that ACEi halted mesangial cell hyperplasia, induced glomerular endothelial cell remodeling, and promoted glomerular cell repopulation [10]. In the same study, lisinopril even increased the number of podocytes compared to the numbers in age-matched untreated animals. These data suggested the neoformation of healthy glomerular tissue after ACEi, but the cellular and molecular determinants remained elusive.

Until not long ago, no one would have imagined there were progenitor cells in the kidney, as there are in other organs capable of regeneration, including the liver. Central to this line of research was the discovery [11] of a population of parietal epithelial cells lining Bowman's capsule in the adult rat kidney, expressing the neural cell adhesion molecule (NCAM), a marker of metanephric mesenchyme (table 1) [12]. NCAM was expressed by the majority of claudin $1+$ parietal epithelial cells, together with CD24, a stemness marker of mouse and human kidney. Costaining of NCAM and the podocyte marker WT1 identified 3 distinct cell populations of epithelial origin lining the Bowman capsule of normal rats. They include immature progenitor cells expressing NCAM, transitional cells expressing markers for progenitor cells and podocytes $(\mathrm{NCAM}+\mathrm{WT} 1+)$, and more differentiated epithelial cells, i.e. the parietal podocytes (NCAM-WT1+). $\mathrm{NCAM}+$ parietal epithelial cells exposed in vitro to appropriate medium differentiate into podocytes, confirming their progenitor nature [11]. The parietal epithelial cells of Bowman's capsule are also a reservoir of podocytes [13].

With this in mind, the behavior of NCAM+ parietal epithelial cells was studied in MWF rats with early lesions consisting of bridges between the parietal and visceral epithelia, followed by hyperplastic lesions that eventually evolved into fibrosis. The majority of cells in glomerular lesions were claudin $1+$ parietal epithelial cells of Bowman's capsule, with only a few podocytes. In old MWF animals, claudin $1+$ parietal epithelial cells increased in number at the expense of parietal podocytes, and they actively proliferated and accumulated in Bowman's space, reflecting a dysregulation of their ability to differentiate into podocytes and repair injury. ACEi limited the forma- 
Table 1. Progenitor cell populations identified in adult healthy kidneys

\begin{tabular}{llll}
\hline Species & Markers & Localization & First author (year) \\
\hline Human & CD133+CD146+ & Glomerular tuft & Bruno (2009) [28] \\
Human & CD133+CD24+CD106+ & Bowman's capsule & Angelotti (2012) [29] \\
& CD24+CD133+CD106- & Proximal and distal tubules & Lindgren (2011) [30] \\
Human & Aldehyde dehydrogenase CD24+CD133+ & Proximal tubules & Benigni (2011) [11] \\
Rat & NCAM+CD24+ & Bowman's capsule & Gupta (2006) [31] \\
Rat & Oct4+ & Proximal tubules & Maeshima (2003) [32] \\
Rat & BrdU (slowly cycling cells) & Proximal and distal tubules & Challen (2006) [33] \\
Mouse & Sca-1+CD24+ (side population) & Proximal tubules & Hishikawa (2005) [34] \\
Mouse & Musculin+MyoR+ (side population) & Interstitium & Plotkin (2006) [35] \\
Mouse & CD34+Sca-1+CD44+ & Interstitium & Pippin (2013) [36] \\
Mouse & Renin & Juxtaglomerular compartment & Oliver (2009) [37] \\
Rat/mouse & BrdU/Histone2B (slowly cycling cells) & Papilla & \\
\hline
\end{tabular}

tion of hyperplastic lesions, preventing the accumulation of extracellular matrix and evolution towards glomerulosclerosis. The reestablishment of a normal glomerular architecture by ACE inhibition was associated with a reduced activation of NCAM+ progenitors and restoration of their distribution along the parietal epithelium of Bowman's capsule. The mechanism by which ACEi foster glomerular regeneration has been ascribed to $\mathrm{C} / \mathrm{EBP} \delta$, a transcription factor specific for adult cells and mitotically quiescent stem cells of epithelial origin which regulates the cell cycle and self-renewal. In MWF rats, the high proliferative status of Bowman's capsule cells demonstrated by the scant expression of the cell cycle inhibitor C/EBP $\delta$ was reversed by ACEi, indicating a path through which the drug directly limits the activation of parietal progenitor cells [11].

Although RAS inhibitors are the current gold standard for renoprotective therapies, their effectiveness greatly depends on when the treatment is started, to the extent that renoprotection was imperfect when therapy was given in the advanced phase of the disease. Every year, some patients with overt proteinuria and worsening kidney function continue to progress to end-stage kidney disease (ESKD) or die prematurely of myocardial infarction or stroke despite RAS inhibitor therapy. This prompted us to study the effectiveness of multimodal intervention strategies targeting pathogenic pathways other than Ang II to achieve renoprotection. Antagonists that bind the receptors of endothelin 1, a major mediator of renal disease progression, have been found to be renoprotective [4]. ACEi combined with endothelin type $\mathrm{A}\left(\mathrm{ET}_{\mathrm{A}}\right)$ receptor antagonist normalized proteinuria and the number of podocytes and provided complete protection from tubulointerstitial damage, while in rats with overt diabetic nephropathy each drug alone gave only partial renoprotection [14]. Combined therapy caused glomerular lesions to regress to levels lower than before the start of treatment, similar to those in controls. A similar effect was obtained by combining maximal RAS inhibition by ACEi plus ARB with a statin in rats with overt diabetic nephropathy. In these animals, the statin lowered the proteinuria to control levels and ameliorated glomerulosclerosis, even inducing regression [15].

These results reinforce the concept that targeting proteinuria is the best way to induce the regression of renal disease, and they suggest a strategy for proteinuric diabetic and nondiabetic patients who do not fully benefit from ACE inhibition and ARB treatment.

\section{Evidence of Kidney Repair in Humans}

The ability of ACEi to halt the progression of proteinuric chronic renal disease has been described in patients with nondiabetic and diabetic nephropathies. Robust evidence of a renoprotective effect comes from the Ramipril Efficacy in Nephropathy (REIN) study where, compared to non-ACEi therapy, ramipril halved the rate of renal function loss and reduced progression to ESKD in patients with nondiabetic chronic nephropathies [16]. Renoprotection was dependent on the duration of the treatment, and in patients receiving continued ramipril therapy for at least 5 years the rate of GFR decline progressively improved [17]. In 10 of these patients [17], there was a key point indicating the transition from an initial phase of substantial protection from renal function 
loss to a second phase of progressive improvement in renal function. There was a sustained increase in GFR with time, suggesting a concomitant improvement in the pathology underlying the regression of glomerulosclerosis that could possibly be linked to some repair and/or regeneration of the kidney. Wilmer et al. [18] reported that treatment with ACEi for 8 years stabilized kidney function in patients with type 1 diabetes and nephrotic syndrome expected to progress to ESKD. That human kidneys can be repaired was demonstrated long ago in patients with type 1 diabetes in whom the typical lesions of diabetic glomerulopathy improved during 10 years of normoglycemia achieved by pancreas transplantation [19].

Renal lesions in diabetes take a long time to develop and to regress, and years of renoprotective treatment are needed before any clinical improvement in renal disease can be seen (which, anyway, occurs in a minority of patients). ACEi at dosages higher than those recommended for blood pressure control, in combination with an ARB and a diuretic, helped improve the clinical outcome, as was first shown in a single individual with rapidly worsening renal function [20]. Later on, multimodal therapy and ACEi alone were formally compared in a large cohort of patients with severe CKD [21]. Over a 7-year followup, only 2 out of 56 patients on the integrated protocol progressed to ESKD compared to 17 out of 56 reference patients. Moreover, the GFR was stabilized in 26 patients, and this was taken to indicate remission of the disease and the possibility of kidney repair in this population [21]. This therapeutic program has been formally named Remission Clinic and is currently implemented at least to some extent even in 'emerging' countries [22] where dialysis and transplantation are simply not available to most patients.

How the kidney can regenerate in response to renoprotective agents and which cells are implicated have been the subjects of recent studies. A key observation was that parietal epithelial cells lining Bowman's capsule expressing stem cell markers CD133 and CD24 are indicated as progenitor cells in the human kidney and give rise to podocytes and tubular cells (table 1) [23]. We recently observed [24] that glomerular hyperplastic lesions in human proliferative glomerulonephritis were derived from the dysregulated proliferation and migration of progenitor cells in Bowman's space in response to an inflammatory stimulus. The abnormal behavior of CD133+CD24+ cells could be the result of podocyte activation. In proliferative disorders the expression of CXCR4, the receptor of the chemokine SDF-1, was high-
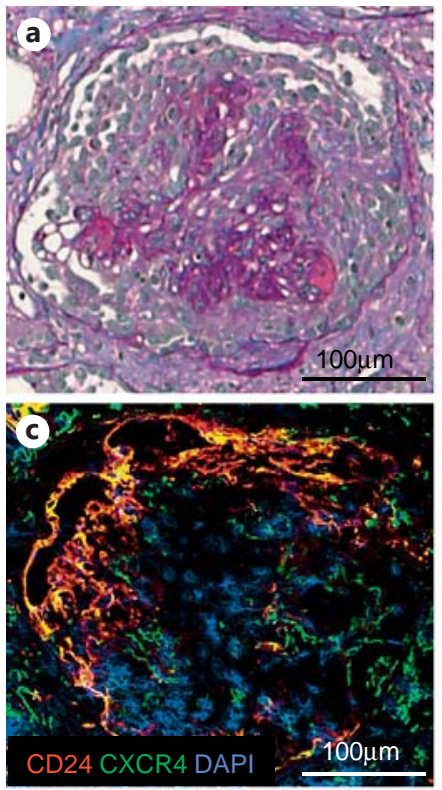

After treatment
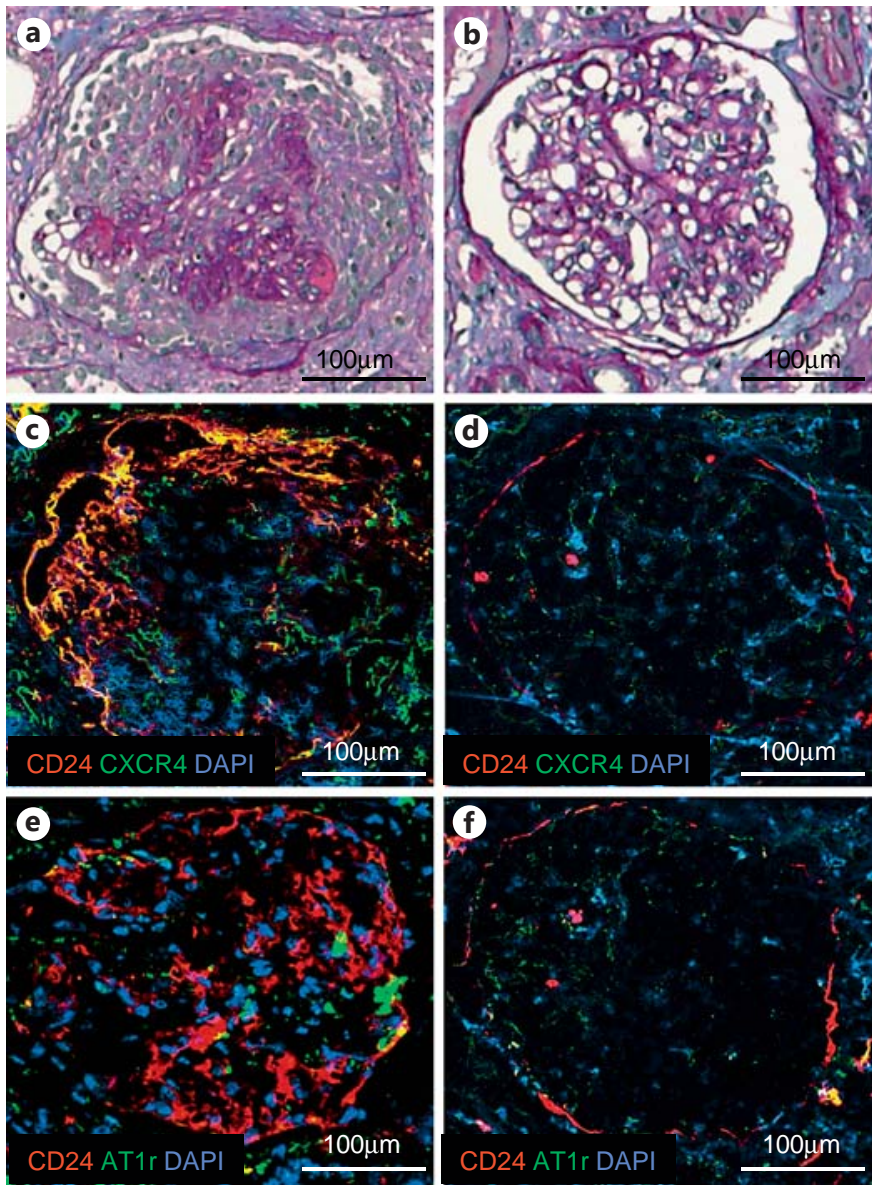

Fig. 1. ACE inhibition reduced glomerular lesions, limited progenitor cell migration, and restored CXCR4/AT 1 receptor expression in a patient with extracapillary glomerulonephritis. a, b PASstained biopsy before treatment indicated that the majority of glomeruli were affected by extracapillary proliferation. After ACEi, all glomeruli were totally free of lesions as shown in the representative glomerulus. c Representative picture of double staining for CD24 (red) and CXCR4 (green) showing that the majority of CD24+ progenitor cells inside the glomerular lesions also expressed CXCR4. d ACEi reduced CXCR4 expression in CD24+ progenitor cells vs. levels before treatment. e Double immunofluorescence staining for $\mathrm{CD} 24$ (red) and $\mathrm{AT}_{1}$ receptor (green) showing $\mathrm{AT}_{1}$ receptor overexpression in progenitor cells in the area of the lesions. $\mathbf{f}$ After ACEi treatment, AT1 receptor expression was weak and no cells in the Bowman capsule coexpressed the CD24 progenitor marker. Nuclei were stained with DAPI (blue).

er in progenitor cells forming the hyperplastic lesions. On the other hand, podocytes activated by the inflammatory environment produced SDF-1, providing the ligand for CXCR4 receptors upregulated on progenitor cells, ultimately allowing their migration and proliferation
94

Nephron Exp Nephrol 2014;126:91-96 DOI: $10.1159 / 000360675$
Gagliardini/Benigni 
[24]. The inflammatory environment is also sustained by Ang II which, through the $\mathrm{AT}_{1}$ receptor, increases the production of reactive oxygen species, cytokines, and adhesion molecules. The $\mathrm{AT}_{1}$ receptor was weakly expressed in progenitor cells of the Bowman capsule and its expression increased in patients with proliferative disorders, suggesting that the Ang/ $\mathrm{AT}_{1}$ receptor pathway may contribute to the abnormal behavior of renal progenitors in hyperplastic lesion formation. ACEi therapy given to a patient with crescentic glomerulonephritis limited progenitor cell proliferation and normalized CXCR4 and $\mathrm{AT}_{1}$ receptor expression on renal progenitor cells, concomitant with the regression of hyperplastic lesions (fig. 1).

These findings suggest that targeting CXCR4/Ang II/ $\mathrm{AT}_{1}$ receptor pathogenic pathways might be beneficial in severe glomerular proliferative disorders [24].

\section{Conclusion}

In recent decades, much progress has been made in organ regeneration thanks to new investigative techniques and more sophisticated research tools. Formerly, reductions in renal damage and the regression of lesions were documented by simple histological observations in two dimensions [6]. Nowadays, recent instruments including microcomputed tomography permit the observation of tissue structures in three dimensions, which greatly facilitates visualization of the spatial distribution and connectivity of microvessels in organs including the kidney. This technique demonstrated improvements in the renal microvascular architecture after chronic $\mathrm{ET}_{\mathrm{A}}$ blockade in pigs with hypercholesterolemia [25] and in stenotic kidneys after infusion with hepatocyte growth factor [26]. Three-dimensional analysis has taught us that bidimensional observation no longer provides enough information on structural changes during the course of the disease or after pharmacological therapy. In a recent study, treatment with an $\mathrm{ET}_{\mathrm{A}}$ receptor antagonist normalized the podocyte number with no significant decrease in proteinuria in animals with glomerulonephritis. Three-dimensional scanning electron microscopy showed that podocytes only partially recovered primary and secondary interdigitating processes after treatment, which would explain their defective function [27]. This highlighted the importance of combining three-dimensional morphological and morphometric analyses to actually visualize podocyte damage/recovery and estimate the degree of protection provided by a given drug.
Identification of progenitor cells in the adult kidney was another step forward in regenerative medicine, suggesting the repair potential of the adult kidney also in mammals. Many progenitor cell populations are located in different areas of the adult kidney (table 1), and the migration of renal precursors into the damaged region might contribute to regeneration. However, reconstitution of functional tissue could theoretically be confined to the early phase of the disease, becoming limited as the damage increases. In the advanced phases, renal progenitors chaotically proliferate and migrate, paving the way to lesion formation and subsequent sclerosis. ACE inhibition influences renal progenitor cell proliferation and migration and restores the glomerular architecture.

Understanding how renal progenitors are maintained and regulated - by characterizing the gene expression profiles and the corresponding signaling molecules could enable us to identify new therapeutic targets. Genetic lineage-tracing approaches will facilitate progenitor cell characterization and enable us to examine their differentiation potential in vivo. These combined efforts will favor the translation of experimental results into clinical practice. Finally, finding the genes associated with the regression of CKD and favorable outcomes will provide a basis for the development of therapeutic strategies for patients with severe renal disorders, and possibly also to overcome cardiovascular complications.

\section{Acknowledgements}

We are grateful to Dr. Paola Rizzo for her helpful assistance, and to J.D. Baggott for English language editing. Manuela Passera helped prepare the manuscript. This work was partially supported by a European Commission grant (project STELLAR No. HEALTH-F4-2012-305436).

References

1 Just PM, Riella MC, Tschosik EA, Noe LL, Bhattacharyya SK, de Charro F: Economic evaluations of dialysis treatment modalities. Health Policy 2008;86:163-180.

2 Gridelli B, Remuzzi G: Strategies for making more organs available for transplantation. $\mathrm{N}$ Engl J Med 2000;343:404-410.

- 3 Dinneen SF, Gerstein HC: The association of microalbuminuria and mortality in non-insulin-dependent diabetes mellitus: a systematic overview of the literature. Arch Intern Med 1997;157:1413-1418.

4 Perico N, Benigni A, Remuzzi G: Present and future drug treatments for chronic kidney diseases: evolving targets in renoprotection. Nat Rev Drug Discov 2008;7:936-953. 
5 Benigni A, Morigi M, Remuzzi G: Kidney regeneration. Lancet 2010;375:1310-1317.

-6 Remuzzi A, Gagliardini E, Sangalli F, Bonomelli M, Piccinelli M, Benigni A, Remuzzi G: ACE inhibition reduces glomerulosclerosis and regenerates glomerular tissue in a model of progressive renal disease. Kidney Int 2006; 69:1124-1130.

7 Ma LJ, Nakamura S, Whitsitt JS, Marcantoni C, Davidson JM, Fogo AB: Regression of sclerosis in aging by an angiotensin inhibitioninduced decrease in PAI-1. Kidney Int 2000; 58:2425-2436.

8 Boffa JJ, Lu Y, Placier S, Stefanski A, Dussaule JC, Chatziantoniou C: Regression of renal vascular and glomerular fibrosis: role of angiotensin II receptor antagonism and matrix metalloproteinases. J Am Soc Nephrol 2003; 14:1132-1144.

\$9 Adamczak M, Gross ML, Krtil J, Koch A, Tyralla K, Amann K, Ritz E: Reversal of glomerulosclerosis after high-dose enalapril treatment in subtotally nephrectomized rats. J Am Soc Nephrol 2003;14:2833-2842.

10 Macconi D, Sangalli F, Bonomelli M, Conti S, Condorelli L, Gagliardini E, Remuzzi G, Remuzzi A: Podocyte repopulation contributes to regression of glomerular injury induced by ACE inhibition. Am J Pathol 2009;174:797807.

-11 Benigni A, Morigi M, Rizzo P, Gagliardini E, Rota C, Abbate M, Ghezzi S, Remuzzi A, Remuzzi G: Inhibiting angiotensin-converting enzyme promotes renal repair by limiting progenitor cell proliferation and restoring the glomerular architecture. Am J Pathol 2011; 179:628-638.

-12 Abbate M, Brown D, Bonventre JV: Expression of NCAM recapitulates tubulogenic development in kidneys recovering from acute ischemia. Am J Physiol 1999;277:F454-F463.

-13 Appel D, Kershaw DB, Smeets B, Yuan G, Fuss A, Frye B, Elger M, Kriz W, Floege J, Moeller MJ: Recruitment of podocytes from glomerular parietal epithelial cells. J Am Soc Nephrol 2009;20:333-343.

14 Gagliardini E, Corna D, Zoja C, Sangalli F, Carrara F, Rossi M, Conti S, Rottoli D, Longaretti L, Remuzzi A, Remuzzi G, Benigni A: Unlike each drug alone, lisinopril if combined with avosentan promotes regression of renal lesions in experimental diabetes. Am J Physiol Renal Physiol 2009;297:F1448-F1456.

-15 Zoja C, Corna D, Gagliardini E, Conti S, Arnaboldi L, Benigni A, Remuzzi G: Adding a statin to a combination of ACE inhibitor and $\mathrm{ARB}$ normalizes proteinuria in experimental diabetes, which translates into full renoprotection. Am J Physiol Renal Physiol 2010; 299:F1203-F1211.

-16 Randomised placebo-controlled trial of effect of ramipril on decline in glomerular filtration rate and risk of terminal renal failure in proteinuric, non-diabetic nephropathy. The GISEN Group (Gruppo Italiano di Studi Epidemiologici in Nefrologia). Lancet 1997;349: 1857-1863.
17 Ruggenenti P, Perna A, Benini R, Bertani T, Zoccali C, Maggiore Q, Salvadori M, Remuzzi G: In chronic nephropathies prolonged ACE inhibition can induce remission: dynamics of time-dependent changes in GFR. Investigators of the GISEN Group. Gruppo Italiano Studi Epidemiologici in Nefrologia. J Am Soc Nephrol 1999;10:997-1006.

18 Wilmer WA, Hebert LA, Lewis EJ, Rohde RD, Whittier F, Cattran D, Levey AS, Lewis JB, Spitalewitz S, Blumenthal S, Bain RP: Remission of nephrotic syndrome in type 1 diabetes: long-term follow-up of patients in the Captopril Study. Am J Kidney Dis 1999;34:308-314.

19 Fioretto P, Steffes MW, Sutherland DE, Goetz FC, Mauer M: Reversal of lesions of diabetic nephropathy after pancreas transplantation. N Engl J Med 1998;339:69-75.

20 Ruggenenti P, Brenner BM, Remuzzi G: Remission achieved in chronic nephropathy by a multidrug approach targeted at urinary protein excretion. Nephron 2001;88:254-259.

-21 Ruggenenti P, Perticucci E, Cravedi P, Gambara V, Costantini M, Sharma SK, Perna A, Remuzzi G: Role of remission clinics in the longitudinal treatment of CKD. J Am Soc Nephrol 2008;19:1213-1224.

22 Limesh M, Annigeri RA, Mani MK, Kowdle PC, Rao BS, Balasubramanian S, Seshadri R: Retarding the progression of chronic kidney disease with renin angiotensin system blockade. Indian J Nephrol 2012;22:108-115.

23 Sagrinati C, Netti GS, Mazzinghi B, Lazzeri E, Liotta F, Frosali F, Ronconi E, Meini C, Gacci M, Squecco R, Carini M, Gesualdo L, Francini F, Maggi E, Annunziato F, Lasagni L, Serio M, Romagnani S, Romagnani P: Isolation and characterization of multipotent progenitor cells from the Bowman's capsule of adult human kidneys. J Am Soc Nephrol 2006;17: 2443-2456.

24 Rizzo P, Perico N, Gagliardini E, Novelli R, Alison MR, Remuzzi G, Benigni A: Nature and mediators of parietal epithelial cell activation in glomerulonephritides of human and rat. Am J Pathol 2013;183:1769-1778.

25 Chade AR, Krier JD, Textor SC, Lerman A, Lerman LO: Endothelin-A receptor blockade improves renal microvascular architecture and function in experimental hypercholesterolemia. J Am Soc Nephrol 2006; 17:3394-3403.

26 Stewart N, Chade AR: Renoprotective effects of hepatocyte growth factor in the stenotic kidney. Am J Physiol Renal Physiol 2013; 304:F625-F633.

27 Buelli S, Rosanò L, Gagliardini E, Corna D, Longaretti L, Pezzotta A, Perico L, Conti S, Rizzo P, Novelli R, Morigi M, Zoja C, Remuzzi G, Bagnato A, Benigni A: $\beta$-Arrestin-1 drives endothelin-1-mediated podocyte activation and sustains renal injury. J Am Soc Nephrol 2014;25:523-533.
28 Bruno S, Bussolati B, Grange C, Collino F, di Cantogno LV, Herrera MB, Biancone L, Tetta C, Segoloni G, Camussi G: Isolation and characterization of resident mesenchymal stem cells in human glomeruli. Stem Cells Dev 2009; 18:867-80.

29 Angelotti ML, Ronconi E, Ballerini L, Peired A, Mazzinghi B, Sagrinati C, Parente E, Gacci M, Carini M, Rotondi M, Fogo AB, Lazzeri E, Lasagni L, Romagnani P: Characterization of renal progenitors committed toward tubular lineage and their regenerative potential in renal tubular injury. Stem Cells 2012;30:17141725.

30 Lindgren D, Boström AK, Nilsson K, Hansson J, Sjölund J, Möller C, Jirström K, Nilsson E, Landberg G, Axelson H, Johansson ME: Isolation and characterization of progenitorlike cells from human renal proximal tubules. Am J Pathol 2011;178: 828-837.

-31 Gupta S, Verfaillie C, Chmielewski D, Kren S, Eidman K, Connaire J, Heremans Y, Lund T, Blackstad M, Jiang Y, Luttun A, Rosenberg ME: Isolation and characterization of kidney-derived stem cells. J Am Soc Nephrol 2006; 17:3028-3040.

-32 Maeshima A, Yamashita S, Nojima Y: Identification of renal progenitor-like tubular cells that participate in the regeneration processes of the kidney. J Am Soc Nephrol 2003; 14: 3138-3146.

33 Challen GA, Bertoncello I, Deane JA, Ricardo SD, Little MH: Kidney side population reveals multilineage potential and renal functional capacity but also cellular heterogeneity. J Am Soc Nephrol 2006; 17: 1896-1912.

34 Hishikawa K, Marumo T, Miura S, Nakanishi A, Matsuzaki Y, Shibata K, Ichiyanagi T, Kohike H, Komori T, Takahashi I, Takase O, Imai $\mathrm{N}$, Yoshikawa $\mathrm{M}$, Inowa $\mathrm{T}$, Hayashi $\mathrm{M}$, Nakaki T, Nakauchi H, Okano H, Fujita T: Musculin/MyoR is expressed in kidney side population cells and can regulate their function. J Cell Biol 2005; 169:921-928.

35 Plotkin MD, Goligorsky MS: Mesenchymal cells from adult kidney support angiogenesis and differentiate into multiple interstitial cell types including erythropoietin-producing fibroblasts. Am J Physiol Renal Physiol 2006;291:F902-F912.

- 36 Pippin JW, Sparks MA, Glenn ST, Buitrago S, Coffman TM, Duffield JS, Gross KW, Shankland SJ: Cells of renin lineage are progenitors of podocytes and parietal epithelial cells in experimental glomerular disease. Am J Pathol 2013; 183:542-557.

37 Oliver JA, Klinakis A, Cheema FH, Friedlander J, Sampogna RV, Martens TP, Liu C, Efstratiadis A, Al-Awqati Q: Proliferation and migration of label-retaining cells of the kidney papilla. J Am Soc Nephrol 2009;20:2315-2327. 